\title{
The Electrical Properties of the Cryolithic Zone of the North-East Asia on the Radioimpedance Soundings Data in the VLF-LF-MF Bands
}

\author{
Yu. B. Bashkuev ${ }^{1, *}$, V. P. Melchinov ${ }^{2}$, M. G. Dembelov ${ }^{1}$, L. Kh. Angarkhaeva ${ }^{1}$, D. G. Buyanova ${ }^{1}$ \\ ${ }^{1}$ Institute of Physical Materials Science of the Siberian Branch of the Russian Academy of Sciences, Ulan-Ude, 670047, Russia \\ ${ }^{2}$ M.K. Ammosov North-Eastern Federal University, Yakutsk, 677980, Russia \\ *Corresponding Author: ludang@rambler.ru
}

Copyright (C) 2013 Horizon Research Publishing All rights reserved.

\begin{abstract}
Results of the measurements of the cryolithic zone electrical properties by the radio impedance sounding method are presented. The surface impedance and geoelectric section of the Arctic permafrost have been determined. The experiments have been performed near Cherskii in the continental zone. The map of geoelectric sections of the cryolithic zone, which takes into account the layered structure of rocks, is constructed.
\end{abstract}

Keywords Cryolithic Zone, Electrical Properties, Surface Impedance

\section{Introduction}

Electromagnetic processes and phenomena in the cryolithic zone were studied in Russia, USA, Canada, Japan, Denmark, and other states. The review of the literature indicated that the number of experimental works devoted to permafrost electrical properties under the in situ occurrence conditions is, on the whole, very small. The interest to the processes of LF-MF radio propagation at high latitudes is both fundamental and practical. Frequency dispersion of permafrost electrical properties $(\sigma$ and $\varepsilon)$ and the consideration of this dispersion in radio propagation processes are of the greatest interest among fundamental phenomena. A practical interest has been aroused by the quality requirements for operation of the radio systems of navigation, direction-finding, and management of the sea, river, and air transport at high latitudes. These requirements are prescribed in the Federal Special-Purpose Program "Global Navigation System". In particular, MF radio beacons $(283-325 \mathrm{kHz})$ with an operating zone of 300-500 $\mathrm{km}$ are intended to transmit differential corrections from satellite navigation systems. These radio beacons will be widely used along northern rivers and on the Arctic coast. The study is also actual because the operation quality of the communication and navigation facilities used to support flights at high latitudes decreases under the action of different inhomogeneities of the underlying Earth's surface, which negatively affects flight safety. The studies have become even more significant in connection with the development of the Arctic Ocean natural resources; ice patrol of the Northern Sea Route; organization of high-latitude polar stations; and development of international cross-polar transeastward routes, part of which passes outside the zone of radar control in the region of North Pole and in the Siberian mountain regions difficult of access. As a result of a new development of the navigation and communication systems in the Russian Arctic sector, it becomes necessary to study spectral characteristics of electromagnetic wave attenuation in a wide frequency band in the cryolithic zone. Such studies make it possible to estimate the effect of permafrost on the quality of radio signal transmission and reception for communication and navigation, which is of prime importance in the Russian Arctic regions.

An analysis of the publications devoted to studying electric properties of the underlying medium and surface wave propagation at high latitudes indicated that the electric properties of the cryolithic zone and the propagation conditions have been studied insufficiently. Reference [1] shows that the effective conductivity $\left(\sigma_{\text {eff }}\right)$ varies within $0.7-2.6 \mathrm{mS} \mathrm{m}^{-1}$ for radio paths between $60^{\circ}-80^{\circ} \mathrm{N}$ and $60^{\circ}-130^{\circ} \mathrm{E}$. The effective conductivity is much higher than the rock electric conductivity obtained by the method of vertical electric sounding (VES) using direct current. Signals at a frequency of $100 \mathrm{kHz}$ were used in measurements. For comparison, we present the map, constructed for north-eastern Russia based on VES data [2], where the permafrost conductivity is $\sigma=0.0025-0.167 \mathrm{mS} \mathrm{m}^{-1}$. A difference in the frozen rock conductivity at frequencies of $10-1000 \mathrm{kHz}$ and direct current reaches one-two and more orders of magnitude. Zakharenko et al [3] also noted that the conductivity of frozen loose and hard rocks in the LF-MF bands is $0.75-3.1 \mathrm{mS} \mathrm{m}^{-1}$. The generalized geoelectric section (GES) model with the following parameters was 
proposed for the Taimyr Peninsula:

$$
\begin{gathered}
\rho_{1}=200 \Omega \mathrm{m} ; \mathrm{h}_{1} \text { is up to } 10 \mathrm{~m} ; \\
\rho_{2}=70 \Omega \mathrm{m} ; \mathrm{h}_{2} \text { is up to } 100 \mathrm{~m} ; \\
\rho_{3}=800 \Omega \mathrm{m} ; \mathrm{h}_{3}=\infty .
\end{gathered}
$$

A disadvantage of the data presented in $[1,3]$ consists in that the effective conductivity $\left(\sigma_{\text {eff }}\right)$ corresponds only to the measured frequency and does not reflect the frozen sequence GES structure. As a result, it is difficult to calculate propagation conditions in a frequency band. From the data presented in [3] it also follows that radio wave measurements using the radio comparison method do not make it possible to determine GES parameters at radio frequencies.

\section{Objective and Methods}

The aim of the present work is to determine the local electrical characteristics of the underlying cryogenic medium at high latitudes in the VLF-LF-MF radio bands. In the measurements we used the method of determining the normalized surface impedance $(\delta)$ of the underlying medium. This impedance is determined as the ratio of the tangential components of the electric $\left(\mathrm{E}_{\tau}\right)$ and magnetic $\left(\mathrm{H}_{\tau}\right)$ fields at the air-ground interface: $\delta=\mathrm{E}_{\tau} /\left(\mathrm{H}_{\tau} \mathrm{Z}_{0}\right)$, where $\mathrm{Z}_{0}=120 \pi$ is the free space impedance. The model of the underlying medium at a local point is considered as a horizontally layered medium with the frequency-independent (or dependent) parameters $\left(\rho_{i}, \varepsilon_{i}\right)$ in each layer of thickness $\mathrm{h}_{i}$. The values of the impedance modulus $(|\delta|)$ and phase $\left(\varphi_{\delta}\right)$ were directly measured using the radio impedance sounding method (RISM) or were calculated based on the GES parameters $\left(\rho_{i}, \varepsilon_{i}, h_{i}\right)$, where $\rho_{i}$ is the electrical resistivity (ER) of the $i$ th layer, and $\varepsilon_{i}$ is the permittivity of this layer.

\section{Surface Impedance and Permafrost GES}

Good convergence of the frequency dependences of the surface impedance modulus $|\delta|$ and $\varphi_{\delta}$ in the band 10-1000 $\mathrm{kHz}$, obtained using the RISM and VES methods, was established in the course of the experimental works performed under different geological and geophysical conditions in southern Siberia and European Russia. This circumstance made it possible to widely use VES archived data to construct prediction maps of GES parameters for these territories [4]. Cryolithic zone GESs are very complicated because loose frozen sediments include horizons of different resistivity due to variable lithology and granulo-metric composition, appearance of interstratal ice, and variable groundwater salinity due to the presence and motion of overpermafrost and intrapermafrost waters. Permafrost GESs are characterized by:

- $\quad$ variable electric properties in horizontal and in depth due to variable lithology and (mainly) temperature, ice content, and cryogenic structure;

- $\quad$ sharp or gradient electric boundaries related only to temperature regime and independent of lithology;

- $\quad$ abrupt (sometimes by several orders of magnitude) decrease in ER in the layer of annual temperature fluctuations during a short summer period, when positive temperatures are formed in the surface layer of thickness $0.3-3 \mathrm{~m}$;

sharp (a factor of 2-10 000) increase in rock ER during freezing and subsequent gradual increase in ER with increasing negative temperatures;

- increase in ER in the zone of annual thermal exchange of thickness 10-30 m, which can be either sharp in the upper part of this zone at temperatures of $\left(-1^{\circ}\right)-\left(-5^{\circ}\right) \mathrm{C}$ or gradual (gradient) most frequently in the lower part of this zone, where temperature varies from $-1^{\circ}$ to $-10^{\circ} \mathrm{C}$.

It has been established [5] that the convergence of the impedance frequency dependences, obtained by the RISM and VES methods, is incomplete for perennially frozen rocks in the cryolithic zone. The calculated (based on VES data) impedance modulus values are always larger than the values measured using RISM by a factor of $1.5-2$, and the impedance phase difference is $20^{\circ}-30^{\circ}$ in the entire frequency band. A rather good coincidence of the calculated (VES) and measured (RISM) $|\delta|$ and $\varphi_{\delta}$ values is reached when resistivity of the surface frozen layer decreases by a factor of 3-10 and more as compared to VES data. Numerous synchronous RISM and VES measurements in the cryolithic zone allowed us to make an important physical conclusion that the ER frequency dispersion between the direct current and VLF-LF frequencies is considerable in the layer of loose frozen sediments.

The surface impedance was measured using RISM in the VLF (17.4, 21.1, 22.3, $23.4 \mathrm{kHz}), \mathrm{LF}(257 \mathrm{kHz})$, and MF $(529 \mathrm{kHz})$ bands at 23 observation sites near Cherskii on the left and right banks of the Kolyma River. The measurements were performed using an IPI-1000 impedance meter, which makes it possible to determine the impedance modulus and phase with errors of $\pm 5 \%$ and $\pm\left(2-3^{\circ}\right)$, respectively. The entire region under study is located in the cryolithic zone since the lower temperature limit of permafrost $\left(-12^{\circ}\right)-\left(-14^{\circ}\right)$ was registered near the Arctic coast in Tiksi [6]. Typical complexes of frozen sedimentary and crystalline rocks, the Kolyma River water area, and tundra lakes were distinguished. On the whole, the impedance modulus $(|\delta|)$ and phase $\left(\varphi_{\delta}\right)$ are highly variable, which indicates that the geoelectric conditions are substantially different in the region of measurements. Table 1 presents the extreme and average values of the impedance modulus and phase for the tundra (left bank) and mountain (right bank) regions. Table 1 indicates that the rock electric properties are very different in the studied regions. The tundra zone is characterized by low values of the impedance modulus and by strongly inductive values of the impedance phase in the VLF-LF bands. For the

upland, the impedance phase acquies weakly inductive values, and the impedance modulus is considerably higher than for the tundra due to a high ER of hard rocks. The surface impedance modulus $|\delta|$ in the band $17-529 \mathrm{kHz}$ changes from 0.005 to 0.24 , i.e., by a factor of 48 . For a fixed frequency, the $|\delta|_{\max } /|\delta|_{\min }$ ratio is $5.2-15.6$. The minimal values of the impedance modulus $\left(|\delta|_{\min }=0.005-0.046\right)$ were 
obtained for the tundra lake water area; the maximal values $\left(|\delta|_{\max }=0.076-0.24\right)$, for crystalline rocks. At all points of measurements, the surface impedance phase is inductive and changes from $-76^{\circ}$ to $-13^{\circ}$. With increasing frequency, the impedance modulus increases and its phase shifts in the weakly inductive region. Thus, strongly inductive $\left(\varphi_{\delta}<-45^{\circ}\right)$ values are observed in $68 \%$ of cases at a frequency of 17.4 $\mathrm{kHz}$ and only in $23 \%$ of cases at a frequency of $529 \mathrm{kHz}$.

The interpretation of the radio impedance sounding in the VLF-MF bands consisted in solving the inverse problem based on the regularization method developed by A. N. Tikhonov [5]. We now consider the results of determining the cryolithic zone GESs. According to the VES data [7], the direct current resistivity of frozen sand and silty clay is $1-80$ $\mathrm{k} \Omega \mathrm{m}$ and sometimes reach 1-2 $\mathrm{M} \Omega \mathrm{m}$. The results of interpretation at radio frequencies indicate that the rock resistivity along the wide band of lowland Arctic coasts is considerably lower. Table 2, Fig. 1A and Fig. 1B present the results of interpreting the frequency dependences of the surface impedance in the studied cryolithic zone.

Table 1. Surface impedance of the cryolithic zone in the VLF-MF bands

\begin{tabular}{|c|c|c|c|c|}
\hline \multirow{2}{*}{ Frequency, $\mathrm{kHz}$} & \multicolumn{2}{|c|}{ Left bank } & \multicolumn{2}{|c|}{ Right bank } \\
\cline { 2 - 5 } & $|\delta|$ & $\varphi_{\delta}$ & $|\delta|$ & $\varphi_{\delta}$ \\
\hline \multirow{2}{*}{17.4} & $\frac{0.005-0.024}{0.011}$ & $\frac{\left(-76^{\circ}\right)-\left(-54^{\circ}\right)}{-63^{\circ}}$ & $\frac{0.021-0.078}{0.036}$ & $\frac{\left(-57^{\circ}\right)-\left(-34^{\circ}\right)}{-49^{\circ}}$ \\
\hline \multirow{2}{*}{257} & $\frac{0.037-0.12}{0.07}$ & $\frac{\left(-67^{\circ}\right)-\left(-47^{\circ}\right)}{-54^{\circ}}$ & $\frac{0.1-0.185}{0.136}$ & $\frac{\left(-50^{\circ}\right)-\left(-15^{\circ}\right)}{-31^{\circ}}$ \\
\hline \multirow{2}{*}{529} & $\frac{0.05-0.184}{0.11}$ & $\frac{\left(-53^{\circ}\right)-\left(-34^{\circ}\right)}{-45^{\circ}}$ & $\frac{0.12-0.24}{0.16}$ & $\frac{\left(-29^{\circ}\right)-\left(-13^{\circ}\right)}{-19^{\circ}}$ \\
\hline
\end{tabular}

Table 2. Parameters of cryolithic zone GESs according to the RISM interpretation data

\begin{tabular}{|c|c|c|c|c|c|c|c|c|c|c|c|c|c|c|}
\hline \multirow{2}{*}{ O.p. no. } & \multicolumn{5}{|c|}{$\rho_{i}, \Omega \mathrm{m}$} & \multicolumn{5}{|c|}{$\varepsilon_{i}$, rel. units } & \multicolumn{4}{|c|}{$\mathrm{h}_{i}, \mathrm{~m}$} \\
\hline & $\rho_{1}$ & $\rho_{2}$ & $\rho_{3}$ & $\rho_{4}$ & $\rho_{5}$ & $\varepsilon_{1}$ & $\varepsilon_{2}$ & $\varepsilon_{3}$ & $\varepsilon_{4}$ & $\varepsilon_{5}$ & $\mathrm{~h}_{1}$ & $\mathrm{~h}_{2}$ & $\mathrm{~h}_{3}$ & $\mathrm{~h}_{4}$ \\
\hline \multicolumn{15}{|c|}{ River and lake water areas } \\
\hline 2 & 200000 & 315 & 8 & 90 & - & 3.2 & 87 & 20 & 20 & - & 0.3 & 10 & 12 & - \\
\hline 3 & 200000 & 330 & 56 & 18 & - & 3.2 & 87 & 20 & 20 & - & 0.3 & 3.5 & 12 & - \\
\hline 6 & 200000 & 63 & 18 & - & - & 3.2 & 87 & 20 & - & - & 0.3 & 4.7 & - & - \\
\hline \multicolumn{15}{|c|}{ Sedimentary rocks, left bank } \\
\hline 4 & 940 & 360 & 110 & - & - & 10 & 10 & 10 & - & - & 5.2 & 37 & - & - \\
\hline 5 & 620 & 11 & - & - & - & 18 & 10 & - & - & - & 24 & - & - & - \\
\hline 8 & 660 & 360 & - & - & - & 10 & 10 & - & - & - & 16 & - & - & - \\
\hline 7 & 200 & 1800 & 320 & 90 & 27 & 10 & 7 & 14 & 10 & 10 & 0.3 & 23 & 56 & 3 \\
\hline \multicolumn{15}{|c|}{ Sedimentary rocks, right bank } \\
\hline 10 & 500 & 1400 & 560 & - & - & 43 & 4 & 10 & - & - & 8 & 58 & - & - \\
\hline 12 & 800 & 1400 & 360 & 200 & - & 10 & 7 & 15 & 10 & - & 0.5 & 18 & 37 & - \\
\hline 16 & 460 & 1250 & 250 & - & - & 10 & 10 & 10 & - & - & 3 & 80 & - & - \\
\hline 18 & 750 & 1670 & 570 & - & - & 10 & 10 & 10 & - & - & 9 & 29 & - & - \\
\hline 23 & 190 & 1240 & 560 & - & - & 14 & 13 & 10 & - & - & 1.4 & 22 & - & - \\
\hline 17 & 1300 & 870 & 310 & - & - & 14 & 10 & 10 & - & - & 36 & 71 & - & - \\
\hline 11 & 2400 & 1000 & - & - & - & 15 & 10 & - & - & - & 37 & - & - & - \\
\hline 14 & 700 & 240 & - & - & - & 40 & 4 & - & - & - & 36 & - & - & - \\
\hline 15 & 1100 & 550 & - & - & - & 20 & 9 & - & - & - & 47 & - & - & - \\
\hline 13 & 500 & 780 & - & - & - & 33 & 16 & - & - & - & 4.7 & - & - & - \\
\hline \multicolumn{15}{|c|}{ Crystalline rocks, right bank } \\
\hline 9 & 780 & 1300 & 1100 & 3200 & - & 35 & 10 & 10 & 10 & - & 14 & 50 & 29 & - \\
\hline 19 & 600 & 6100 & - & - & - & 10 & 10 & - & - & - & 4.5 & - & - & - \\
\hline 20 & 1100 & 9100 & - & - & - & 10 & 10 & - & - & - & 7 & - & - & - \\
\hline 21 & 2400 & 4900 & - & - & - & 10 & 10 & - & - & - & 11 & - & - & - \\
\hline 22 & 3900 & 11200 & - & - & - & 10 & 10 & - & - & - & 70 & - & - & - \\
\hline
\end{tabular}


A
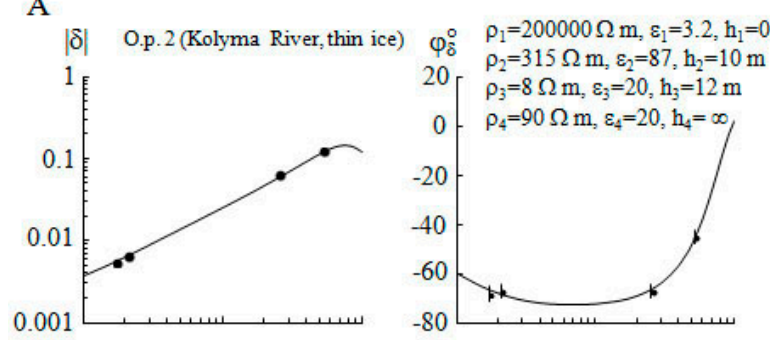

o.p. 3 (Kolyma River, midstream, thin ice)
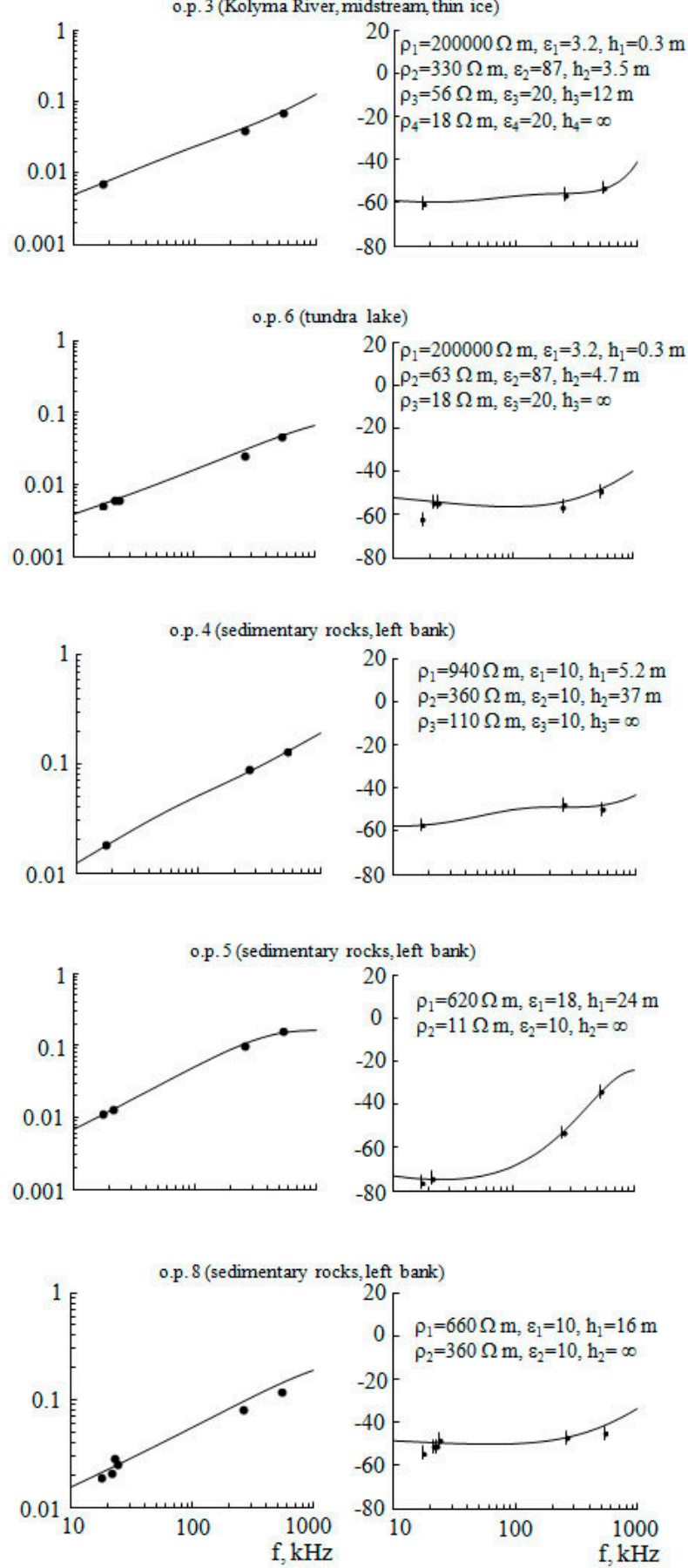

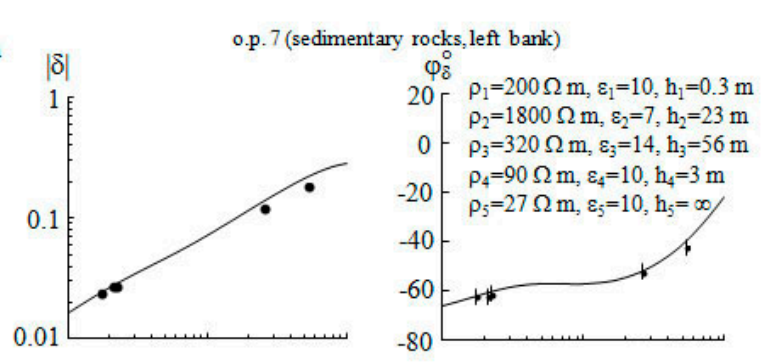

o.p. 10 (sedimentary rocks, left bank)
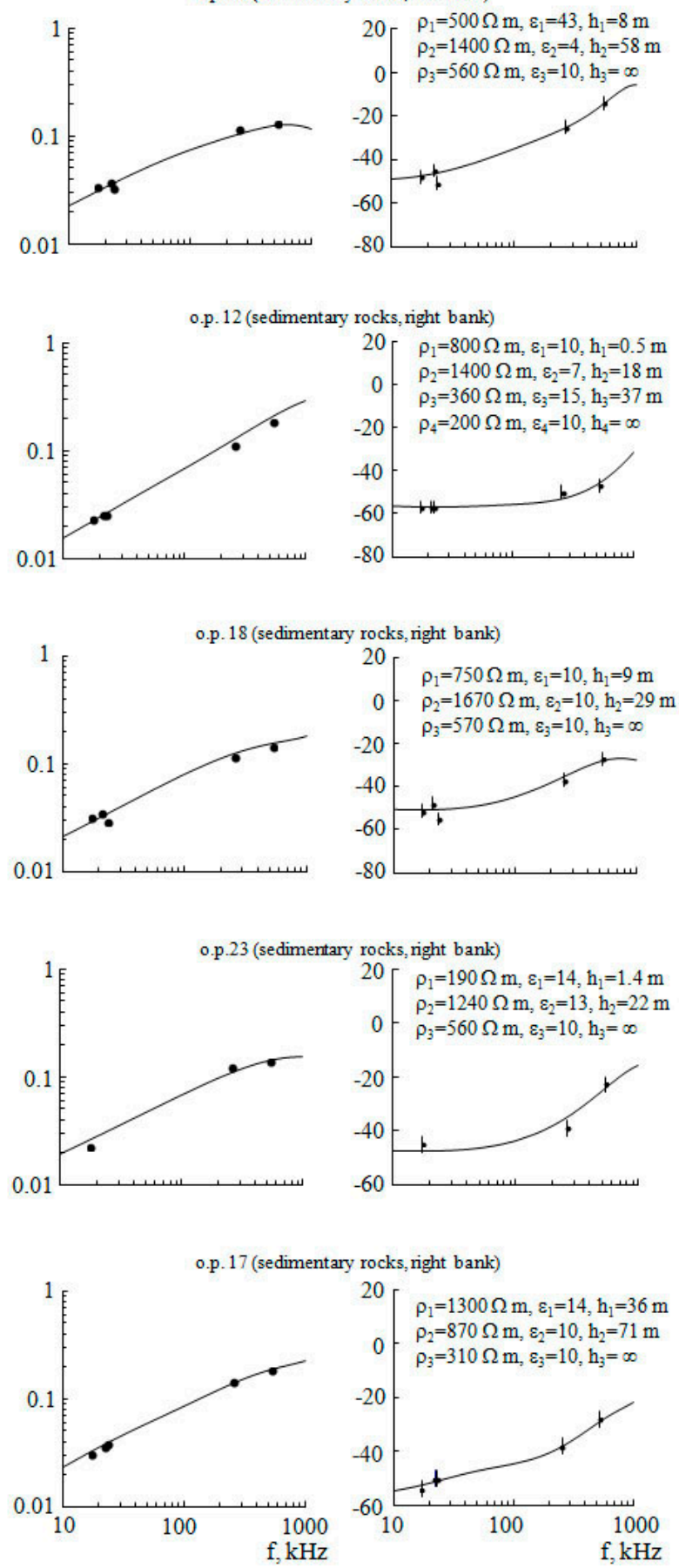

Figure 1. Part A. Results of interpreting the radio impedance soundings near the Arctic coast 

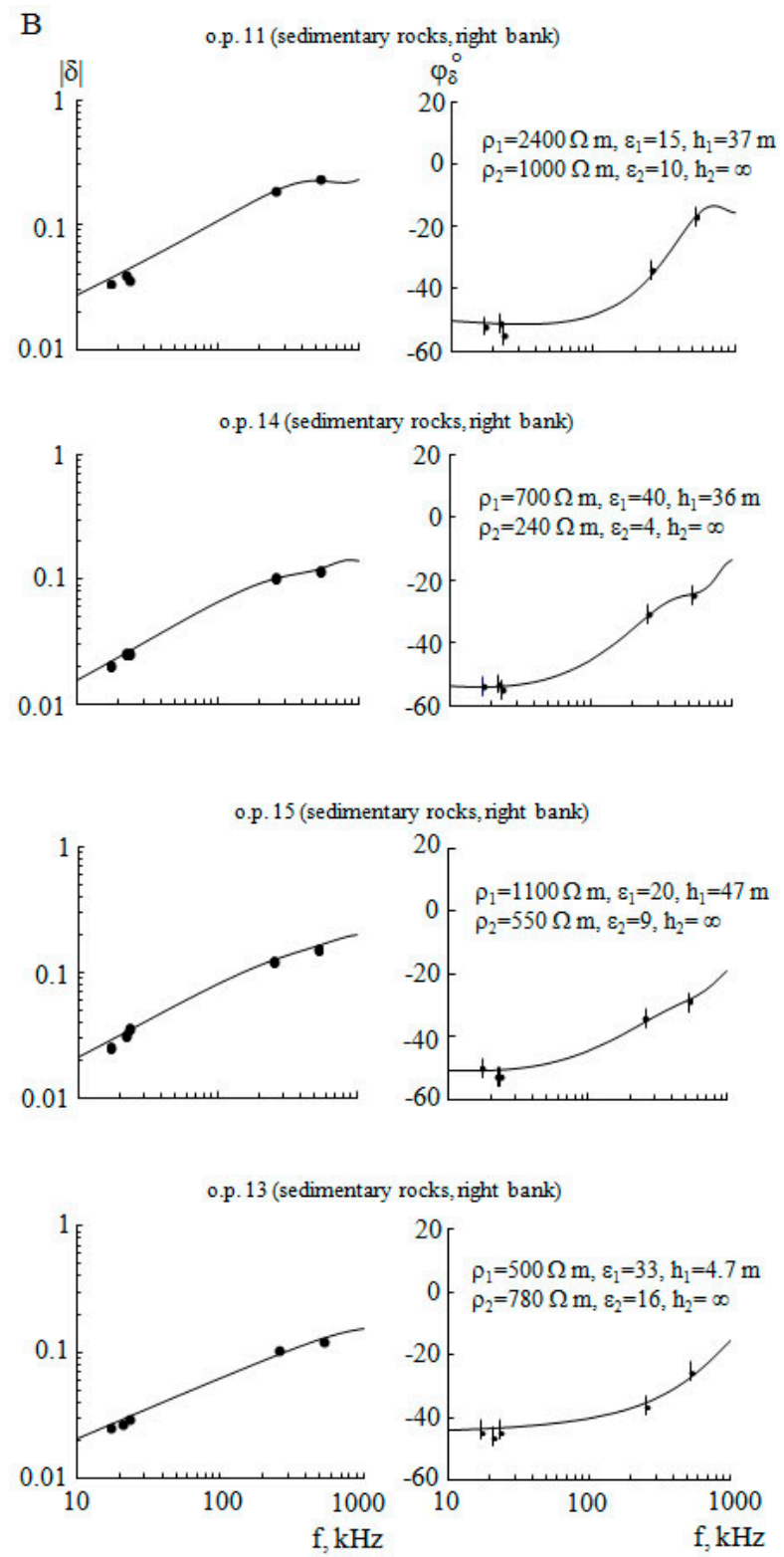
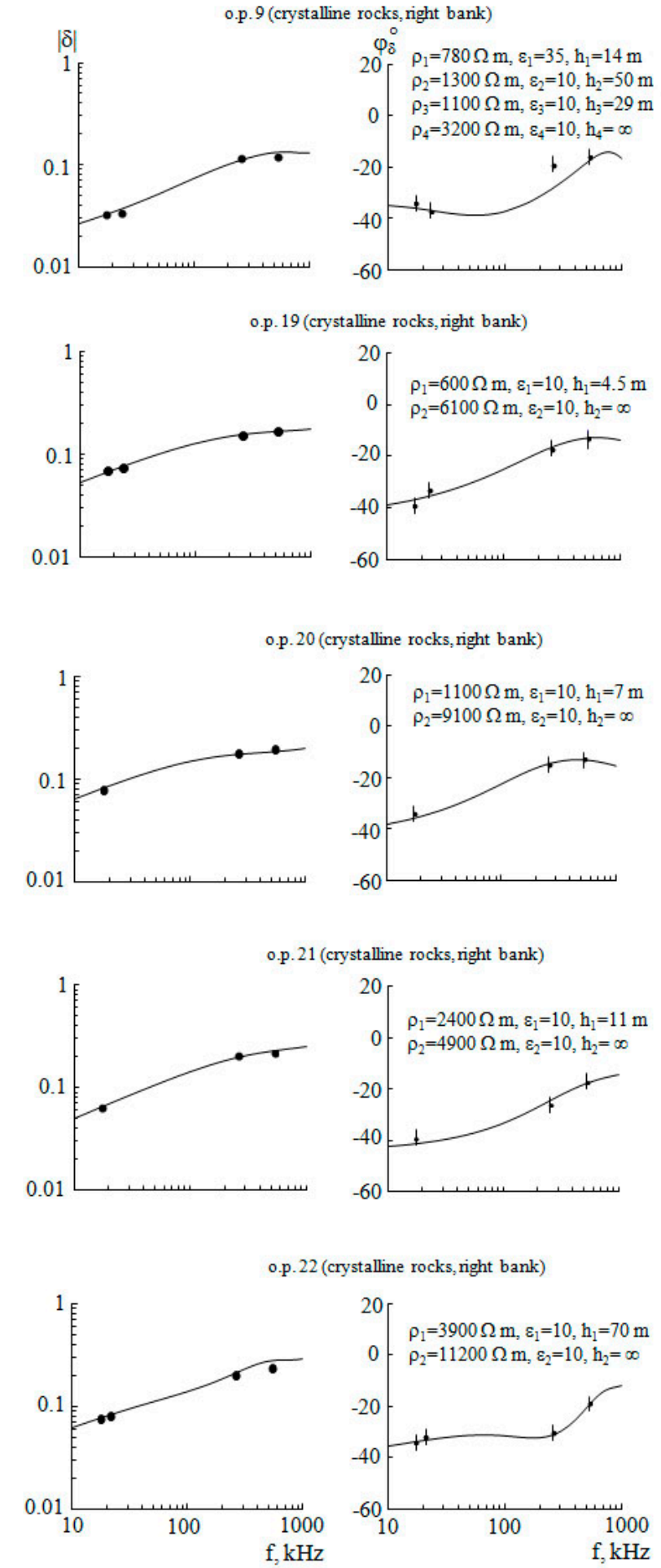

Figure 1. Part B. Results of interpreting the radio impedance soundings near the Arctic coast

For the sedimentary rocks (Figure 1, observation points $4,5,7,8,10-18,23)$, the resistivity of the frozen layer of thickness $18-80 \mathrm{~m}$ changes from 700 to $2400 \Omega \mathrm{m}$, which is considerably lower than the known VES values of $\rho_{i}$. The section of a $\rho_{1}>>\rho_{2}$ type, with a very low resistivity $\left(\rho_{2}=11\right.$ $\Omega \mathrm{m}$ ) of the underlying frozen layer, seems to be most interesting. Such a cryogenic structure is of a cryopeg type and is rather widespread in the cryolithic zone. A low resistivity of the frozen sedimentary sequence (11-60 $\Omega \mathrm{m}$ ) near the Arctic seas can be caused by the penetration of seawater with increased salinity during recent geological epochs.

The sections of crystalline frozen rocks (Figure 1, observation points 9,19-22) are mainly of a $\rho_{1}<\rho_{2}$ type with 
a layer resistivity of $600-11200 \Omega \mathrm{m}$. High resistivity values (from 3200 to $11200 \Omega \mathrm{m}$ ) and the $\rho_{2} / \rho_{1}$ ratio varying from 2 to 10 are typical of monolithic hard rocks with low porosity and insignificant fracturing.

GESs of water areas have been studied. According to the interpretation data, the resistivity of the Kolyma River water is about $300 \Omega \mathrm{m}$ (Table 2, Fig. 1A, observation points 2,3), which is substantially higher than the water resistivity of most rivers in southern Siberia. The tundra lake (Fig. 1A, observation point 6 ) has a water resistivity of about $60 \Omega \mathrm{m}$, which is close to the water resistivity of midlatitude fresh-water lakes. The lake is underlain by more conductive bottom sediments with a resistivity of $18 \Omega \mathrm{m}$. The surface impedance of water areas of northern rivers and lakes depends on a basin depth, electric parameters of water and bottom soil, and electrophysical properties of ice and snow in winter seasons. Basin depths are usually $2-30 \mathrm{~m}$; therefore, in the VLF-MF bands, these basins should be considered as two-layer formations in summer and three- and four-layer formations and in winter. Electric conductivity and temperature coefficient of natural water increase by $2-3 \%$ and $\varepsilon \approx 0.35 \mathrm{deg}^{-1}$, respectively, if temperature increases by $1{ }^{\circ} \mathrm{C}$. Electric conductivity of fresh surface water varies within $(1.6-40) \mathrm{mS} \mathrm{m} \mathrm{m}^{-1}$. Thus, $\sigma=(7.5-10.9)$, (3-4), (11.6-13.9), and (7.7-12.5) $\mathrm{mS} \mathrm{m}^{-1}$ for the Lena River upstream of Yakutsk, the Kolyma River near Cherskii, the Irtysh River, and Lake Baikal, respectively. Generalized GES of the water-bottom soil layered medium can be represented in the form: $\varepsilon_{1}=80, \rho_{1}=20-300 \Omega \mathrm{m}, \mathrm{h}_{1}=2-30$ $\mathrm{m} ; \varepsilon_{2}=20-30, \rho_{2}=10-500 \Omega \mathrm{m}, \mathrm{h}_{2}=\infty$. GES of a $\rho_{1}<\rho_{2}$ type is encountered most frequently; e.g., $\rho_{1}=70-80 \Omega \mathrm{m}$ and $\rho_{2}=200-400 \Omega \mathrm{m}$ for the Selenga River (pebble with sand). On the whole, water areas of rivers and lakes also satisfy the impedance boundary conditions in the VLF-MF bands since $\left|\varepsilon_{\mathrm{k}}\right| \gg 1$ for water. At the same time, these areas are not sharp interfaces and, consequently, insignificantly disturb the amplitude-phase structure of the electromagnetic field.

We now compare the electric properties of the underlying medium for three regions in Yakutia and Transbaikalia, located at distances of about $1800 \mathrm{~km}$ from each other from north to south. The impedance was measured in summer. For the frozen sedimentary rocks in north-eastern Russia, GESs of a $K$ type $\left(\rho_{1}<\rho_{2}>\rho_{3}\right)$ are most typical to a depth of $150 \mathrm{~m}$. Figure 2 illustrates the results of interpreting the radio impedance soundings in the VLF-MF bands at latitudes of $69^{\circ}-52^{\circ} \mathrm{N}$. In the VLF band, the structure of the cryolithic zone to a depth of the skin-layer is of the same type for the tundra regions near the Arctic coast, central Yakutia, and the Vitim Plateau. The resistivity and thickness of the layer of loose frozen sediments in central Yakutia is larger than in the northern and southern parts of the cryolithic zone. The extensive experimental data generally indicate that the resistivity of loose frozen rocks in the VLF-MF bands is lower than the known VES values by 1-3 orders of magnitude. A difference between the cryolithic zone impedance measured in the VLF-LF-MF bands and calculated using the VES data indicated that the RISM data should be used to estimate the GES parameters. Thus, based on the average statistical characteristics $\left(|\delta|\right.$ and $\left.\varphi_{\delta}\right)$ for the cryolithic zone of the Lena-Amga interfluve [8], the generalized three-layer section of a $\rho_{1}<\rho_{2}>\rho_{3}$ type with the following parameters was determined during the interpretation of the impedance frequency dependence in the band $17-864 \mathrm{kHz}$ :

$$
\begin{gathered}
\rho_{1}=7.5 \Omega \mathrm{m} ; \mathrm{h}_{1}=0.1 \mathrm{~m} ; \\
\rho_{2}=1070 \Omega \mathrm{m} ; \mathrm{h}_{2}=42.5 \mathrm{~m} ; \\
\rho_{3}=270 \Omega \mathrm{m} ; \mathrm{h}_{3}=\infty .
\end{gathered}
$$

In summer, obtained GES is typical of the Lena-Amga interfluve where sodic and alkali soils are encountered. The interpretation results also indicate that the RISM resolution with respect to $\rho_{i}$ and $h_{i}$ is high.

\section{Prediction Map of GESs for Northeastern Russia}

The results considered above were used in the geoelectric mapping of the cryolithic zone and in the construction of the GES prediction map of northeastern Russia, the fragment of which is presented in Figure 3. The method for compiling a GES prediction map for the cryolithic zone is based on the relation of rock electric properties to lithology and geocryological conditions [4]. Geological maps and RISM and VES data are used in this case. To reliably estimate GES parameters for the problems of radio propagation, it is necessary to use RISM measurements in geoelectric mapping. In this case the complex value of the surface impedance, used in LF-MF propagation calculations, is directly measured, and all GES parameters $\left(\rho_{i}, \varepsilon_{i}, h_{i}\right)$ are determined when the problems of inverse radio sounding are solved [5]. If the maps of the GES parameters constructed based only on VES data are used to calculate the LF-MF field, the erroneous conclusion that radio wave attenuation above paths in the cryolithic zone is considerable can be drawn. Moreover, using the VES method, it is principally impossible to estimate permittivity of layers $\left(\varepsilon_{i}\right)$, which plays a substantial role in the regions of occurrence of perennially frozen ground with low conductivity. The problem of measuring permittivity of in situ frozen soils is still insufficiently studied. We constructed the map of GES parameters for the cryolithic zone based on the results of direct measurements of the surface impedance modulus and phase. After recalculations, the GES map changes into the surface impedance map, which is used to calculate the attenuation function (W) and the field levels in a wide band of radio waves. The presence of forest, surface ice, and seasonally thawing layer on a radio path is taken into account on the GES map by introducing an additional layer. 

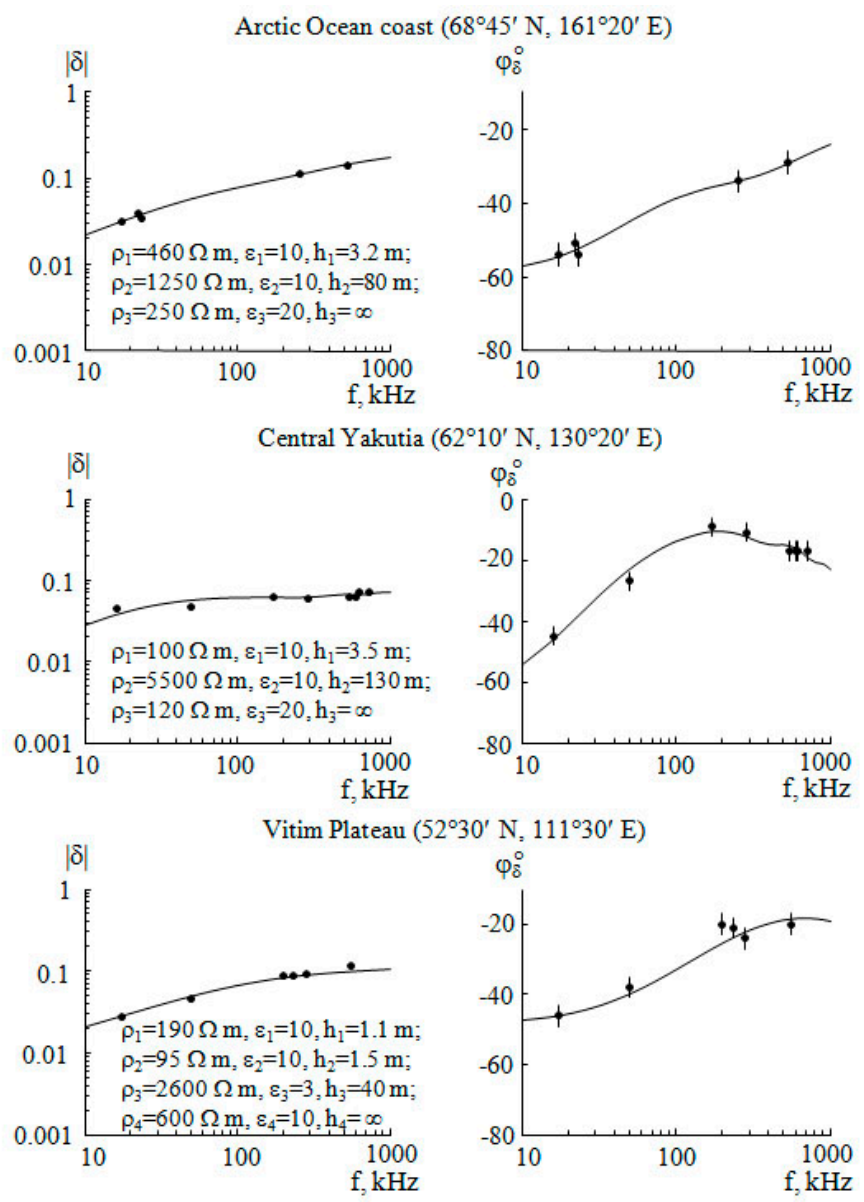

Figure 2. Frequency dependences of the surface impedance and the parameters of the permafrost GES at different latitudes

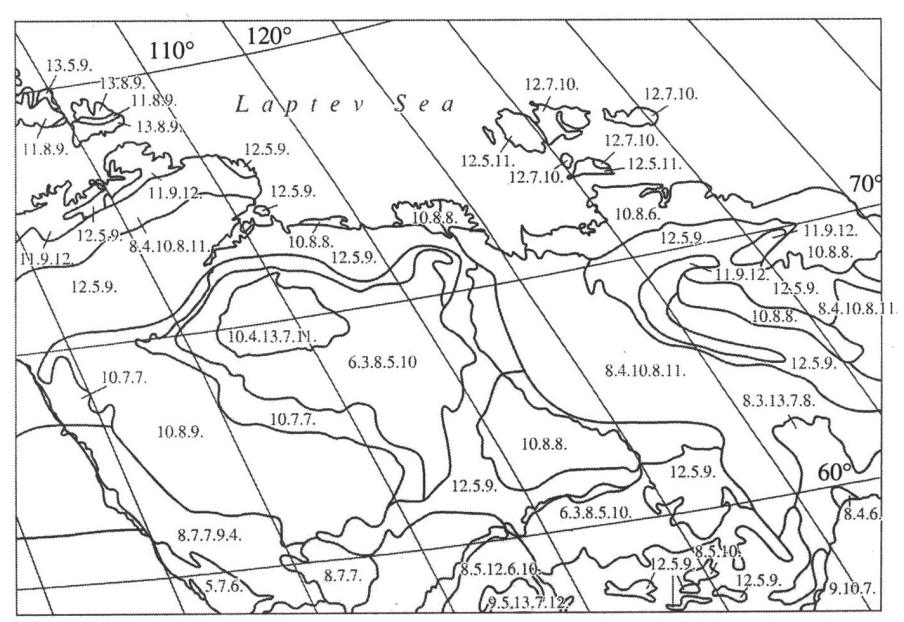

\begin{tabular}{|c|c|c|}
\hline Step no. & $\begin{array}{c}\text { Median } \rho_{i} \text { value, } \\
\Omega \mathrm{m}\end{array}$ & Median $h_{i}$ value, $\mathrm{m}$ \\
\hline 4 & 14.7 & 1.47 \\
5 & 31.6 & 3.16 \\
6 & 68.0 & 6.8 \\
7 & 147 & 14.7 \\
8 & 316 & 31.6 \\
9 & 680 & 68 \\
10 & 1470 & 147 \\
11 & 3160 & 316 \\
12 & 6800 & 680 \\
13 & 14700 & 1470 \\
\hline
\end{tabular}

Figure 3. Fragment of the prediction map of GESs for northeastern Russia 


\section{Conclusion}

GES of the cryolithic zone and the surface impedance of different-type perennially frozen rocks were determined as a result of the complex radio-geophysical studies. The results of studying the electric properties of the cryogenic mediums in the VLF-LF-MF bands resulted in the creation of the new generation of the maps of cryolithic zone electric properties, which take into account the layered structure of rocks and have no analogs in other states. The GES maps make it possible to increase the accuracy of electromagnetic field calculations by a factor of 1.5-3 as compared to such an accuracy obtained using the available Morgan-Maxwell map. The GES maps can form the basis for constructing digital GIS maps which can be widely applicable in high-accuracy radio systems. The value of the performed studies consists in that new experimental data were obtained as a result of the unique ground-based experiments performed at high latitudes.

\section{Acknowledgements}

This article was prepared with the partial financial support of RFBR, grant numbers 12-02-98002, 12-01-98010, 12-05-98051 and SB RAS integration project number 11.

\section{REFERENCES}

[1] B. A. Korobov, V. P. Lantsov, B. G. Neuimin, T. I. Sergienko, O.V. Shtykov. Studying Effective Electric Properties of the Underlying Surface at High Latitudes, Kilometer Wave Propagation, Ed. by M. I. Beloglazov, Kola Fil. Akad. Nauk SSSR, Apatity, Russia, 85-87, 1987.

[2] V. S. Yakupov, S. G. Byalobzheskii, Sh. Sh. Gasanov, L. I. Izmaylov, G. V. Lapshin. Map of Rock Electric Conductivity in the Permafrost Zone of the Northeastern USSR, Fizika Zemli, No.8, 102-104, 1969.

[3] V. N. Zakharenko, L. N. Popov, I. A. Vyltsan, V. M. Sidekhmenova. Electric Conductivity of the Underlying Earth's Surface in the Zone of High Latitudes, Geomagn. Aeron, Vol.29, No.2, 347-349, 1989.

[4] Yu. B. Bashkuev, V. R. Advokatov, L. Kh. Angarkhaeva. Maps of Geoelectric Sections in the Eastern Hemisphere, Fizika Zemli, No.9, 87-94, 2003.

[5] L. Kh. Angarkhaeva, Yu. B. Bashkuev, V. P. Melchinov. Inverse Problem for a Layered Impedance Medium, Radiotekh. Elektron., Vol.42, No.10, 1169-1173, 1997.

[6] A. A. Ogilvie. Engineering Geophysics, Nedra, Moscow, Russia, 1988.

[7] V. S. Yakupov. Electric Conductivity and the Geoelectric Section of Frozen Rocks, Nauka, Moscow, Russia, 1968.

[8] V. P. Melchinov, A. I. Kuzmin. Surface Impedance in the Lena-Amga Interfluvial Region in the VLF-LF Range, Electromagnetic Wave Propagation, BF SO Akad. Nauk SSSR, Ulan-Ude, Russia, 111-119, 1987. 